\title{
Pallor and Stroke-Like Symptoms in a Three-Year-Old Child
}

\author{
Kasi A. Eastep, ${ }^{\mathrm{a}, \mathrm{b}}\left(\mathbb{D}\right.$, Sandra Herr ${ }^{\mathrm{a}}$
}

\begin{abstract}
Acute lymphocytic leukemia (ALL) is the most common childhood malignancy and can include a vast array of symptoms at the time of diagnosis. This case describes a 3-year-old child who presented with worsening pallor, immobility and fatigue who was found to have papilledema and focal neurological findings on physical examination. An emergent diagnostic workup revealed severe anemia. The patient's neurological deficits improved after administration of oxygen and gentle intravenous fluid resuscitation. He was ultimately diagnosed with B-cell ALL; a combination of leukocytosis/leukostasis in the microcirculation and severe anemia were thought to have contributed to this child's unique presentation.
\end{abstract}

Keywords: Anemia; Cerebral vascular accident; Pediatric; Leukemia

\section{Introduction}

Acute lymphocytic leukemia (ALL) has a peak incidence at 2 - 5 years of age and is the most common childhood malignancy, affecting 3.4 per 100,000 children each year in the United States [1]. ALL often presents with a collection of symptoms, most notably anorexia, fatigue, malaise, pallor, irritability, low-grade fever, bone pain with or without limp, bruising, bleeding, petechiae, hepatosplenomegaly, lymphadenopathy, and/or painless testicular enlargement/mass [2]. Symptoms of childhood leukemia are usually the result of bone marrow replacement and subsequent cytopenias or extramedullary infiltration of leukemic blast cells into various tissues [3].

Hyperleukocytosis, often defined as a white blood cell (WBC) count greater than $100,000 / \mu \mathrm{L}$, is an oncologic emergency that can result in mental status change, headache, seizure activity, and papilledema due to leukocytosis in the microcirculation, resulting in hypoperfusion to critical tissues. It is recommended that platelets be transfused to maintain a count above

Manuscript submitted July 29, 2021, accepted November 29, 2021

Published online December 31, 2021

aNorton Children's Affiliated With the University of Louisville School of Medicine, Department of Pediatrics, Division of Pediatric Emergency Medicine, Louisville, KY, USA

${ }^{\mathrm{b}}$ Corresponding Author: Kasi Eastep, Division of Pediatric Emergency Medicine, Department of Pediatrics, University of Louisville, Louisville, KY 40202, USA. Email: kasi.eastep@1ouisville.edu

doi: https://doi.org/10.14740/ijcp451
200,000/mL; red blood cell transfusions should be avoided, if possible, as this will lead to increased viscosity. Hydration, alkalization and allopurinol are key interventions when hyperleukocytosis is suspected, and disease-directed treatment should be initiated once clinical stability has been achieved. If central nervous or pulmonary symptoms exist, leukapheresis or exchange transfusion can be considered to reduce the WBC count prior to administering cytotoxic therapy [2].

\section{Case Report}

A previously healthy 3-year-old male patient presented to our emergency department after progressive worsening of fatigue and pallor that began approximately 2 weeks prior to arrival. The child was ambulatory with assistance, but he became less interactive/mobile on the afternoon of presentation, prompting evaluation. There was no associated fever, emesis or decrease in urine output. He was seen by his pediatrician 1 week prior, but no diagnostic workup was performed at that time. His vaccinations were up-to-date, there were no known allergies, and he did not take any daily medication(s). His parents both had a history of iron-deficiency anemia. The child was noted to be a "picky eater", but it was determined that he would eat items from all food groups upon further questioning. His parents denied excessive milk intake.

The patient arrived with a temperature of $36.6{ }^{\circ} \mathrm{C}$, heart rate of 164 beats per minute, blood pressure of $98 / 50 \mathrm{~mm}$ $\mathrm{Hg}$, respiratory rate of 32 breaths per minute and had a pulse oximetry of $100 \%$ in room air. Physical exam findings were significant for extreme pallor, bilateral papilledema, left conjugate gaze palsy, flaccid left upper extremity, weak left lower extremity (strength of hip and knee flexion 4 out of 5), sustained ankle clonus of the left lower extremity, and a child who was only able to answer questions with one-word responses.

The patient was brought to the resuscitation bay where oxygen was immediately administered via a nonrebreather facemask. An i-STAT (point of care venous blood gas) was obtained given the concern for anemia and quickly revealed a $\mathrm{pH}$ of 7.43, $\mathrm{pCO}_{2}$ of $35 \mathrm{~mm} \mathrm{Hg}, \mathrm{pO}_{2}$ of $50.1 \mathrm{~mm} \mathrm{Hg}$ (with supplemental oxygen) and a hemoglobin of $2.3 \mathrm{~g} / \mathrm{dL}$. Given the initial concern for hyperleukocytosis, a $10 \mathrm{~mL} / \mathrm{kg}$ normal saline bolus was slowly administered. A computed tomography (CT) of the head without contrast was emergently obtained and was unremarkable. After initiation of supplemental oxygen and intravenous fluids, the patient had gradual improvement in the strength of his left upper extremity with limited fine motor ability and resolution of his left conjugate gaze palsy. His speech also became more comprehensible, and he was able to 


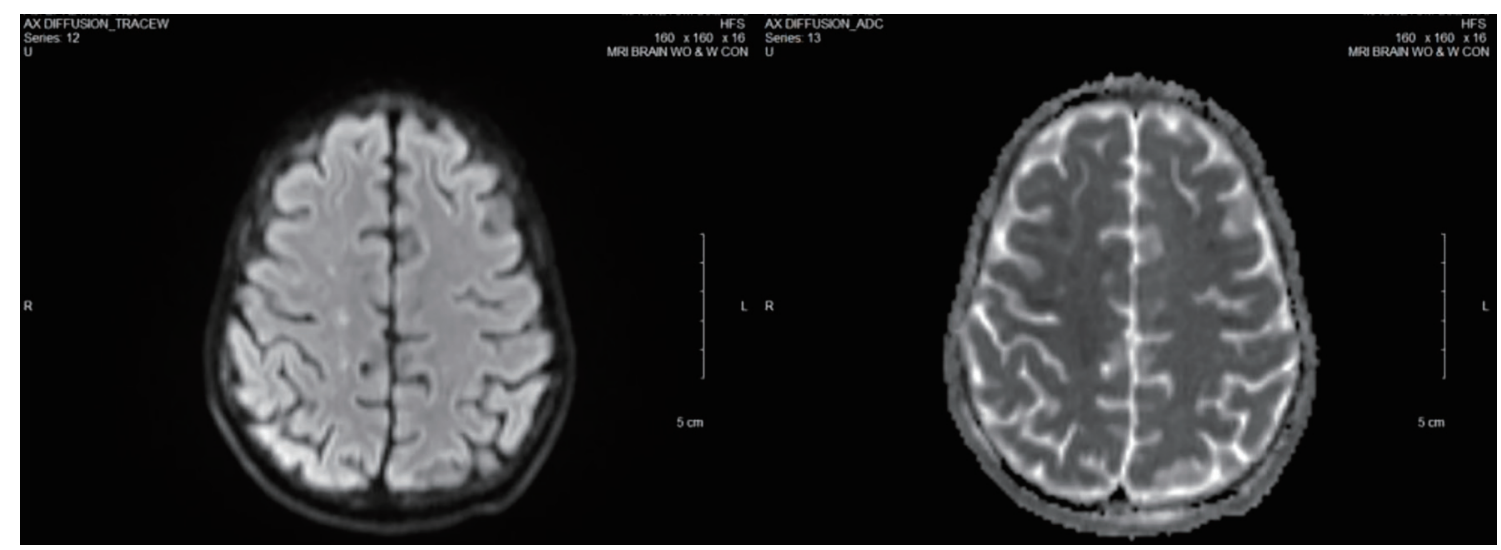

Figure 1. Patient's magnetic resonance imaging (MRI) of the brain, diffusion-weighted imaging (DWI), left, versus apparent diffusion coefficient (ADC) map, right. The DWI sequence on the left shows a few, scattered punctate areas of diffusion restriction in the right centrum semiovale. There is not clear corresponding ADC signal. These were thought to be small areas of ischemic change versus edema, not active central nervous system (CNS) leukemia.

use full sentences. Once type-specific, irradiated packed red blood cells became available, a $3 \mathrm{~mL} / \mathrm{kg}$ transfusion was started prior to admission to the intensive care unit. His vitals upon admission were: temperature of $38.2{ }^{\circ} \mathrm{C}$ for which blood cultures were obtained, heart rate of 130 beats per minute, blood pressure of $92 / 62 \mathrm{~mm} \mathrm{Hg}$, respiratory rate of 32 breaths per minute and had a pulse oximetry of $100 \%$ in room air.

The child's initial complete blood count ultimately demonstrated a WBC count of $27,340 / \mu \mathrm{L}$ ( $1 \%$ neutrophils, $20 \%$ lymphocytes and $79 \%$ blasts), hemoglobin of $2.1 \mathrm{~g} / \mathrm{dL}$ (mean corpuscular volume reassuring at $84.4 \mathrm{fL}$, mean corpuscular hemoglobin concentration normal at $32.3 \mathrm{~g} / \mathrm{dL}$ and red cell distribution width within the reference range at $15.5 \%$ ), hematocrit of $6.5 \%$ and a platelet count of $93,000 / \mu \mathrm{L}$. A comprehensive metabolic panel was within normal limits and specifically revealed a blood urea nitrogen of $15 \mathrm{mg} / \mathrm{dL}$, creatinine of $0.3 \mathrm{mg} / \mathrm{dL}$, aspartate aminotransferase of $25 \mathrm{U} / \mathrm{L}$ and an alanine aminotransferase of $14 \mathrm{U} / \mathrm{L}$. His magnesium and phosphorous levels were elevated to 2.6 and $4.8 \mathrm{mg} / \mathrm{dL}$, respectively; lactate dehydrogenase was also elevated to $635 \mathrm{U} / \mathrm{L}$ (reference: 313 - $618 \mathrm{U} / \mathrm{L}$ ), but his uric acid level was within normal limits. A urinalysis, first obtained 3 days later, only demonstrated a low specific gravity to 1.004 . The abovementioned blood culture was ultimately negative.

The patient's follow-up magnetic resonance imaging of the head, obtained once his hemoglobin had improved to 9.0 $\mathrm{g} / \mathrm{dL}$, was significant for foci of hyperintense signal in the right centrum semiovale on the diffusion-weighted sequence but was without a definite corresponding hypointense signal on the apparent diffusion coefficient map (Fig. 1). It was suggested that this finding could represent evolving subacute ischemia/hemorrhage versus nonspecific gliosis/white matter injury. Cerebral spinal fluid (CSF) studies were later obtained; no leukemic cells were identified and his WBC count was $0 /$ $\mu \mathrm{L}$, but the opening pressure was elevated to $36 \mathrm{~cm} \mathrm{H}_{2} \mathrm{O}$ for which Diamox was given. Anticoagulation was held given a reassuring magnetic resonance venography (MRV). He received several small aliquots of packed red blood cells, and his residual weakness slowly resolved over the next several days.

Unfortunately, the patient experienced two spontaneous gastric perforations during induction of chemotherapy that required both gastric resection and gastrostomy tube placement; the surgical specimen did not reveal extensive inflammation upon pathological review, and his gastrostomy tube has since been removed. Today, the patient continues with his antineoplastic therapy but is doing well, overall.

\section{Discussion}

We describe papilledema and transient focal neurological deficits in a child ultimately found to have severe normocytic anemia in the setting of newly diagnosed B-cell ALL. His symptoms dramatically improved with immediate oxygen supplementation and administration of a small fluid bolus. He later had complete resolution of his focal neurological findings after receiving packed red blood cells. This case is unique in that most children with leukemia who experience these symptoms have significant elevation in the WBC count, evidence of central nervous system (CNS) leukemia, or both. A combination of leukocytosis/leukostasis in the microcirculation and severe anemia were thought to have contributed to this child's unusual presentation.

The literature has previously described the rare association between papilledema caused by idiopathic intracranial hypertension and iron-deficiency anemia in adult case reports [4, 5]. The patient described here had an elevated iron level to 271 $\mu \mathrm{g} / \mathrm{dL}$, but this isolated value was obtained shortly following the transfusion of packed red blood cells. In addition, anemia has been categorized as the "fifth cardiovascular risk factor" in adult patients given an increased odds ratio of morbidity, hospitalization and mortality similar to risk factors such as diabetes mellitus and/or smoking [6,7]. Case reports have also described the association between severe iron-deficiency anemia and cerebral venous thrombosis in pediatric patients $[8,9]$. Somewhat similar to our patient, these individuals presented with pallor, vomiting, weakness/alteration in mental status and, in one case, tremulous extremities complicated by right eye deviation. One study has even demonstrated that previously healthy children who experienced a stroke were 10 times 
more likely to have iron-deficiency anemia when compared to their healthy counterparts [10]. While the literature continues to demonstrate an association between iron-deficiency anemia and hypercoagulable events, this patient's normocytic anemia in the setting of circulating blast cells remains curious.

A reassuring MRV in the setting of ongoing neurological deficits renders a thrombotic event highly unlikely; in addition, the child's WBC count was never elevated to a level where CNS effects would be expected from hyperleukocytosis alone. CNS leukemia, defined as a WBC count of $5 / \mu \mathrm{L}$ or more in the CSF with documented lymphoblasts and/or the presence of a cerebral mass or cranial nerve palsy, can result in papilledema, diffuse retinal disease, and cranial nerve palsies; however, our patient did not meet the criteria for this complication [11].

\section{Learning points}

Our case, to the best of our knowledge, represents a rare manifestation of severe normocytic anemia as it relates to ALL. Given this association, we recommend that a complete blood count be considered as part of the diagnostic workup in all children presenting with papilledema and/or focal neurological deficits, especially in the setting of sub-acute fatigue or pallor. Oxygen should rapidly be administered to mitigate hypoxia/ hypoperfusion, even if pulse oximetry is reassuring. One can also consider gentle fluid resuscitation $(5-10 \mathrm{~mL} / \mathrm{kg})$ until blood product becomes available, but should only do so in the setting of frequent clinical reassessment.

\section{Acknowledgments}

None to declare.

\section{Financial Disclosure}

None to declare.

\section{Conflict of Interest}

None to declare.

\section{Informed Consent}

The patient's parents provided written informed consent.

\section{Author Contributions}

Dr. Kasi Eastep drafted the initial manuscript, and reviewed and revised the manuscript. Dr. Sandy Herr reviewed and revised the manuscript.

\section{Data Availability}

The data supporting the findings of this study are available from the corresponding author upon reasonable request.

\section{References}

1. Ward E, DeSantis C, Robbins A, Kohler B, Jemal A. Childhood and adolescent cancer statistics, 2014. CA Cancer J Clin. 2014;64(2):83-103.

2. Engorn B, Flerlage J. The Harriet Lane Handbook, 20th ed. Philadelphia, PA: Saunders; 2015.

3. Shaw KN, Bachur RG, Chamberlain JM, Lavelle J, Nagler J, Shook JE. Fleisher \& Ludwig's Textbook of Pediatric Emergency Medicine. Philadelphia, PA: Wolters Kluwer; 2016.

4. Biousse V, Rucker JC, Vignal C, Crassard I, Katz BJ, Newman NJ. Anemia and papilledema. Am J Ophthalmol. 2003;135(4):437-446.

5. Knizley H, Jr., Noyes WD. Iron deficiency anemia, papilledema, thrombocytosis, and transient hemiparesis. Arch Intern Med. 1972;129(3):483-486.

6. Kaiafa G, Kanellos I, Savopoulos C, Kakaletsis N, Giannakoulas G, Hatzitolios AI. Is anemia a new cardiovascular risk factor? Int J Cardiol. 2015;186:117-124.

7. Spence RK. The economic burden of anemia in heart failure. Heart Fail Clin. 2010;6(3):373-383.

8. Curatola A, Ferretti S, Gatto A, Valentini P, Lazzareschi I. Iron-deficiency anaemia in childhood: a risk factor for severe venous thrombosis? Paediatr Int Child Health. 2021:1-5

9. Habis A, Hobson WL, Greenberg R. Cerebral sinovenous thrombosis in a toddler with iron deficiency anemia. Pediatr Emerg Care. 2010;26(11):848-851.

10. Maguire JL, deVeber G, Parkin PC. Association between iron-deficiency anemia and stroke in young children. Pediatrics. 2007;120(5):1053-1057.

11. Laningham FH, Kun LE, Reddick WE, Ogg RJ, Morris EB, Pui CH. Childhood central nervous system leukemia: historical perspectives, current therapy, and acute neurological sequelae. Neuroradiology. 2007;49(11):873-888. 